\title{
¿Es necesaria la punción lumbar en la evaluación del recién nacido febril sin signos de focalización?
}

\author{
Manuel Díaz Á., Yamilka Viel R., Bárbara Acosta B. y Daniel Claver I.
}

\section{¿Is it necessary to perform a lumbar puncture in the evaluation of the febrile newborn infant without source?}

Lumbar puncture to detect bacterial meningitis is a recommended screening procedure to be performed in febrile newborn infants at admission to hospital. Objective: To evaluate the usefulness of this procedure performed at admission and eventually, in a second look if clinically justified. Patients and method: There were studied 1,841 febrile newborns consulting at the Emergency Department from January 1992 to December 2000 without source. All of them underwent a sepsis workup. The rate of meningitis incidence was calculated, and we analyzed the frequency of meningitis diagnosed in the initial evaluation and reappraisal. Results: There were 206 febrile patients with diagnosis of meningitis for an incidence of $11.2 \%$, which 20 were bacterial (1.1\%) and 186 aseptic meningitis (10.1\%). In the initial evaluation 155 patients were diagnosed with meningitis $(155 / 1.774=8.7 \%)$ and in reappraisal (mean 39,5 $\pm 25,2$ hours after) there were diagnosed another 51 cases $(51 / 135=37.8 \%)$. Conclusions: The meningitis was a common diagnosis in febrile newborns without source, with prevalence of aseptic meningitis. In almost $25 \%$ of cases we reached to this diagnosis by means of performing lumbar puncture after a reappraisal.

Key words: Newborn, lumbar puncture, fever, bacterial meningitis, aseptic meningitis.

Palabras clave: Neonato, punción lumbar, fiebre, meningitis bacteriana aguda, meningitis aséptica.

\section{Introducción}

$\mathrm{E}$ 1 recién nacido $(\mathrm{RN})$ febril sin signos de focalización (SSF) aparente, ha sido siempre un problema de diagnóstico y manejo ${ }^{1,2}$. Cuando se evalúa al RN febril SSF, debemos sospechar una posible infección del SNC, sea de causa bacteriana o viral. La punción lumbar (PL) se efectúa en forma regular en estos casos, siempre que no haya contra-indicaciones $^{3,4}$.

En algunos RNs febriles SSF se puede establecer el diagnóstico de meningitis en la evaluación inicial, pero otras veces el proceso infeccioso está en una etapa muy temprana, evolucionando más tarde a afectarse el SNC y por ello, un examen del LCR normal en la evaluación inicial no es garantía de que no pueda aparecer este foco durante la evolución clínica del proceso infeccioso. Por otra parte, la PL puede haber sido omitida al ingreso del paciente o resultar fallida, sin obtenerse muestra para su análisis.

En nuestra institución, todos los RNs febriles son sometidos a un protocolo de manejo que incluye para la pesquisa de infección ${ }^{5}$, su internación para una observación clínica continua que permite al médico detectar cambios evolutivos desfavorables y le inducen a realizar nuevas evaluaciones del caso. De esta manera, algunos pacientes son reevaluados con una segunda PL pudiendo establecerse el diagnóstico de meningitis en segunda intención.

Todo lo anterior ha despertado el interés de verificar si esta conducta beneficia al paciente en la práctica clínica. El presente estudio tuvo por objeto determinar la incidencia de meningitis en $\mathrm{RN}$ febriles sin foco clínico evidente ponderando la frecuencia de su diagnóstico en la evaluación inicial y en una re-evaluación.

\section{Pacientes y Método}

Se realizó una investigación descriptiva y retrospectiva, cuyo universo de estudio estuvo constituido por todos los pacientes de hasta 30 días de edad, ya egresados de hospitales maternos y provenientes de la comunidad, asistidos consecutivamente en el Servicio de Urgencia por presentar fiebre. Sólo se incluyeron aquellos RNs con síndrome febril agudo que no presentaban signos de focalización en la evaluación inicial y que fueran ingresados en el Servicio de

\author{
Ciudad de la Habana, Cuba \\ Servicio de Neonatología \\ Hospital Pediátrico Universitario \\ Juan M. Márquez \\ Recibido: 25 de abril 2007 \\ Aceptado: 10 de marzo 2008 \\ Correspondencia a: \\ Manuel Díaz Álvarez \\ mfdiaz@infomed.sld.cu
}


Neonatología del Hospital Pediátrico Universitario Juan Manuel Márquez, en el período comprendido entre enero del año 1992 y diciembre del 2000. La investigación fue aprobada por el Consejo Científico del hospital.

Las variables de estudio fueron: sexo, edad gestacional y peso al nacer, número de PLs efectuadas y resultado del LCR en la evaluación inicial y del segundo LCR en la re-evaluación, motivos de la nueva PL, tiempo que medió entre ambos procedimientos y diagnóstico definitivo.

Definiciones. $R N$ febril SSF: aquel que no presentó manifestaciones clínicas focales que explicaran el origen de la fiebre, después de ejecutar el médico un interrogatorio a los familiares y un examen físico al RN en su evaluación inicial. Apariencia tóxico-infecciosa: se definió, por consenso de los médicos de asistencia del Servicio de Neonatología, como un RN que presentaba hiporreflexia, letargia, signos de pobre perfusión periférica y marcada hiperventilación, hipoventilación o cianosis. Fiebre persistente o recurrente: episodios de fiebre posteriores a las 12 horas de ser ingresado. Meningitis aséptica (MA): presencia de manifestaciones clínicas de infección junto con pleocitosis superior a $30 / \mathrm{mm}^{3}$, sin presencia de hematíes en el LCR y necesariamente además, un cultivo bacteriológico de sangre y del LCR negativos. Meningitis bacteriana aguda (MBA): presencia de manifestaciones clínicas de infección junto con pleocitosis del LCR $>30 / \mathrm{mm}^{3}$, con cultivo bacteriológico positivo del LCR y/o sangre.

A todo RN se le realizó una evaluación inicial por médicos especialistas, complementada con exámenes de laboratorio. La PL fue ejecutada por un médico residente de Pediatría en rotación por el Servicio, supervisado siempre por un especialista de Neonatología. A todos los pacientes se les aplicaron los criterios de riesgo de infección bacteriana grave (IBG) creados en nuestra institución para la evaluación de RNs febriles $\mathrm{SSF}^{(6)}$ y se clasificaron en los grupos de riesgo corres-

\begin{tabular}{|c|c|c|c|}
\hline \multicolumn{2}{|c|}{ Variables epidemiológicas } & \multirow{2}{*}{$\begin{array}{l}\mathbf{n} \\
941\end{array}$} & \multirow{2}{*}{$\begin{array}{c}\% \\
51,1\end{array}$} \\
\hline Edad cronológica & $<15$ días & & \\
\hline & $\geq 15$ días & 900 & 48,9 \\
\hline \multirow[t]{2}{*}{ Sexo } & Masculino & 1.038 & 56,4 \\
\hline & Femenino & 803 & 43,6 \\
\hline \multirow[t]{2}{*}{ Edad gestacional } & $<37$ semanas & 21 & 1,1 \\
\hline & $\geq 37$ semanas & 1.820 & 98,8 \\
\hline \multirow[t]{2}{*}{ Peso al nacer } & $<2.500$ gramos & 38 & 2,1 \\
\hline & $\geq 2.500$ gramos & 1.803 & 97,9 \\
\hline
\end{tabular}

pondientes. Si fue necesario, se hicieron re-evaluaciones de los pacientes en las primeras horas de ingresados, si no se tuvieran aún resultados de los cultivos iniciales, o estos hubieran sido negativos.

El uso del tratamiento antibacteriano estuvo sujeto a la consideración del médico que hizo la evaluación inicial, basado en la clasificación de riesgo de IBG. Los ajustes terapéuticos dependieron de la evolución del paciente, los resultados de los cultivos y las re-evaluaciones efectuadas por su médico tratante.

La muestra de LCR fue enviada a análisis convencional citoquímico y bacteriológico.

La realización de una segunda PL fue una decisión clínica de su médico tratante, hubiera sido exitosa o fallida al ingreso. Para el análisis se incluyeron, también, los casos en que no se realizó la PL en la evaluación inicial sino sólo en segunda instancia, con intención de reevaluar al paciente.

Se calcularon la tasa de incidencia de meningitis, tiempo mediano entre la primera y segunda PL y se realizaron pruebas de hipótesis para diferencias de proporciones en el diagnóstico de meningitis entre la evaluación inicial y reevaluación. Consideramos un nivel de significación para $\mathrm{p}<0,05$.

\section{Resultados}

Se estudió un total de 1.841 RNs febriles SSF, de los cuales 1.820 (98,8\%) y 1.803 (97,9\%) fueron $\geq 37$ semanas $\mathrm{y} \geq 2.500$ gramos respectivamente (Tabla 1 ). Hubo un ligero predominio del sexo masculino (1.038 = $56,4 \%)$.

Se detectó una meningitis en 206 RNs, (incidencia de $11,2 \%)$. Veinte pacientes $(1,1 \%)$ tuvieron aislamiento bacteriano en LCR y/o sangre: Streptococcus agalactiae (n: 14) y un aislado de cada uno de los siguientes microorganismos: Pseudomonas aeruginosa, Escherichia coli, Enterobacter sp, Streptococcus pyogenes, Staphylococcus aureus y Flavobacterium sp. En 186 casos (10,1\%) el diagnóstico definitivo fue de MA.

En el proceso de evaluación inicial, se efectuó PL a 1.774 (96,4\%) pacientes y de éstas, fueron fallidas 150 (8,5\%) (Tabla 2). En 67 de los RNs (3,6\%) no se efectuó PL. En 155 casos (8,7\%) el examen citoquímico del LCR fue anormal, por lo que se diagnosticó meningitis, incluyendo de causa bacteriana o MA.

Durante la evolución fue necesario hacer re-evaluación a 16 RNs con PL fallida en la evaluación inicial, resultando con meningitis 43,7\% (todas fueron MA).

De aquellos con un LCR normal inicialmente (1.469) se re-evaluaron 117, detectándos 43 (36,8\%) meningitis (en 8 casos la causa fue bacteriana y el resto MA). 
Tabla 2. La punción lumbar en evaluación inicial y frecuencia de meningitis en recién nacidos febriles sin signos de focalización

\begin{tabular}{lcc|} 
Punción lumbar & $\mathbf{n}$ & $\%$ \\
\hline Realizadas & 1.774 & $96,4 *$ \\
\hline Anormal (meningitis bacteriana) & 12 & $0,7 * *$ \\
\hline Anormal (meningitis aséptica) & 143 & 8,0 ** \\
\hline Fallida & 150 & $8,5 * *$ \\
\hline No realizadas & 67 & $3,6 *$ \\
\hline & & \\
\hline \multirow{2}{*}{$\%$ calculado de $n=1.841, * *$ \% calculado de $n=1.774$} \\
\hline
\end{tabular}

De dos pacientes re-evaluados entre los 67 neonatos en que no se practicó PL en la evaluación inicial, en uno se diagnosticó una MA (Tabla 3).

En general, para todos los casos re-evaluados, se diagnosticaron 37,8\% (51/135) pacientes con meningitis (bacteriana o viral).

El tiempo entre la primera y segunda PL tuvo una media de 39,5 \pm 25,2 horas. Las re-evaluaciones con ejecución de una segunda PL fueron motivadas por algunas manifestaciones en la evolución clínica desfavorables. En los RNs con un LCR normal en la evaluación inicial, los motivos de re-evaluación fueron fiebre persistente o recurrente $(101=86,3 \%)$, estado tóxicoinfeccioso $(14=12,0 \%)$ y otras manifestaciones de evolución adversa $(2=1,7 \%)$. En los casos re-evaluados, que tuvieron una PL fallida en un primer intento durante la evaluación inicial, los motivos que impulsaron a realizar tal re-evaluación fueron: fiebre persistente o recurrente $(12=75 \%$ ) y estado tóxico-infeccioso (4 $=25 \%)$.

\section{Discusión}

Con el diagnóstico definitivo al egreso de los RNs febriles SSF pudimos conocer la incidencia de meningitis que resultó ser de $11,2 \%$, a expensas mayoritariamente de MA, probablemente viral. En otras casuísticas con una casuística similar de RNs o lactantes pequeños febriles SSF la incidencia de MA y MBA se reporta en 3,6 a $19,9 \%^{7-11}$ y 0,3 a $2,4 \%{ }^{10-12}$ respectivamente. Nuestra incidencia se correspondió con estas publicaciones internacionales: aproximadamente 1 de cada 9 RNs febriles SSF tuvo una meningitis, más frecuentemente aséptica (de causa probable viral) y como tal, refuerza el planteamiento de que la ejecución de una PL debe formar parte de la evaluación de los neonatos febriles ${ }^{12-21}$. Nuestra Guía de Prácticas Clíni-
Tabla 3. La punción lumbar en re-evaluación y frecuencia de meningitis en recién nacidos febriles sin signos de focalización

\begin{tabular}{lcccc|}
$\begin{array}{l}\text { Resultado de punción } \\
\text { lumbar en evaluación } \\
\text { inicial }\end{array}$ & $\mathbf{n}$ & $\begin{array}{c}\text { Re- } \\
\text { evaluados }\end{array}$ & $\begin{array}{c}\text { Con } \\
\text { meningitis }\end{array}$ & $\%$ \\
\hline LCR normal & 1.469 & 117 & $43^{*}$ & 36,7 \\
\hline Fallida & 150 & 16 & 7 & 43,7 \\
\hline No realizada & 67 & 2 & 1 & 50,0 \\
\hline Total & 1.686 & 135 & $51 *$ & 37,8 \\
\hline * 8 pacientes con meningitis bacteriana aguda & & & \\
\hline
\end{tabular}

cas en Neonatología ${ }^{22}$ establece esta conducta para los RNs. Diversos trabajos demuestran que en la práctica clínica los RNs febriles SSF son evaluados con mayor frecuencia con una PL, en comparación con lactantes de mayor edad con el mismo cuadro de presentación ${ }^{11,23-24}$.

Pudiera argumentarse que la incidencia de MBA, en la era actual de la prevención por vacunas de infecciones bacterianas tales como Haemophilus influenzae y Streptococcus pneumoniae, o de la profilaxis antimicrobiana intraparto a la mujer embarazada portadora de S. agalactiae, es baja ${ }^{25}$. Sin embargo, no hay dudas que los RNs con sepsis tienen elevada probabilidad de presentar una MBA; así se ha reportado consistentemente que aproximadamente $1 / 4$ de los RNs con sepsis tiene un foco meníngeo ${ }^{26}$. Wiswell y cols ${ }^{27}$, contestan a los temores de ejecutar una PL en RNs con riesgo o sospecha de sepsis, alegando que "este procedimiento diagnóstico toma sólo algunos minutos en realizarse y conocerse los resultados, y que, a pesar de que es baja la incidencia de MBA, ante estas situaciones clínicas, no hay otra vía para documentar la presencia de meningitis".

La meningitis viral tiene, por lo general, un curso muy benigno; no obstante, si no se diagnostica oportunamente, estos neonatos afectados cursarán con fiebre "inexplicable" (habitualmente durante 3 a 5 días), provocando incertidumbre e inquietud -tanto a los familiares como al propio médico tratante- e induciendo muchas veces a la indicación de antibioterapia empírica, prolongando innecesariamente la hospitalización. Por otra parte, la interpretación de un LCR con pleocitosis bajo el efecto del antimicrobiano, resulta más difícil, existiendo el riesgo, ante la duda, de mantener el tratamiento antibacteriano. JL Baraff, artífice de la publicación por un panel de expertos de los lineamientos de manejo del RN y niño febril ${ }^{13}$, igualmente refiere que podría cuestionarse la realización de 
una PL como parte de la evaluación de riesgo en los lactantes febriles bajo 3 meses de edad si se toma en cuenta sólo la incidencia de MBA, pero también hace la observación de que "se ha diagnosticado la MA en este grupo de pacientes evaluados con una PL hasta en $10 \%$ de los casos"28.

Por todo lo anterior, y a nuestro juicio, es necesario realizar una PL como parte de la evaluación de un RN febril SSF.

Un aspecto a considerar cuando se ingresa un RN febril SSF, quien en la evaluación inicial tuvo un examen del LCR normal o la PL resultó fallida, o bien fue omitida, es tener un alto índice de sospecha de la existencia de una meningitis si la evolución clínica del caso no es favorable, e insistir en re-evaluar esta posibilidad. Esto quedó de manifiesto, en nuestra experiencia, en 51 de 135 pacientes, cuya explicación pudiera deberse a que una parte de los RNs son llevados con mucha premura al médico e internados en los inicios del cuadro séptico y antes de constituirse el foco meníngeo. Los 51 RNs identificados con meningitis en la re-evaluación representan $24,7 \%$ de los diagnósticos de meningitis al egreso en esta población de pacientes, destacando la importancia de la cuidadosa observación y realización de una segunda PL si la evolución del paciente no transcurre favorablemente.

La expresión de una evolución clínica desfavorable, motivo de la re-evaluación, se circunscribió a la ocurrencia de fiebre persistente (86,3\%), o la transformación de la apariencia del $\mathrm{RN}$ a un estado tóxico-infeccioso (12\%), parámetros semiológicos que se utilizan en el Servicio de Neonatología para clasificar de alto riesgo al paciente- y vigilarlos continuamente durante toda la estancia del RN en el hospital.

Concluimos que la PL se realizó en casi todos los pacientes, comprobándose que la meningitis fue un diagnóstico frecuente en los RNs febriles SSF procedentes de la comunidad; predominó la MA (probablemente de etiología viral). En algunos pacientes se llegó a este diagnóstico mediante la ejecución de una PL en segunda instancia, por una evolución desfavorable, manifestada principalmente por fiebre persistente o recurrente y/o aspecto tóxico-infeccioso.

Nuestra experiencia en esta serie apoya la recomendación establecida en las Guías de Prácticas Clínicas en Neonatología, de incluir el examen del LCR como parte de la evaluación inicial a todo RN febril SSF y ante una evolución clínica desfavorable -cuando aún no se tiene un diagnóstico- realizar una segunda PL, aun cuando en la evaluación inicial la primera arrojara resultados de LCR normales, no se hubiera realizado una PL o ésta hubiera sido fallida.

\section{Resumen}

La evaluación del RN febril incluye efectuar una punción lumbar para detectar un foco meníngeo. Objetivo: Evaluar la utilidad de este procedimiento al ingreso del paciente al hospital y, eventualmente, en una segunda instancia si se justifica clínicamente. Pacientes y Método: Estudiamos 1.841 recién nacidos febriles sin signos de focalización que consultaron en la Unidad de Emergencia entre enero 1992 y diciembre 2000. Todos se sometieron a una evaluación para pesquisa de sepsis. Calculamos la tasa de incidencia de meningitis, y analizamos la frecuencia de meningitis diagnosticada en la evaluación inicial y en una re-evaluación. Resultados: Hubo 206 pacientes con diagnóstico de meningitis (incidencia de 11,2\%), de las cuales 20 fueron bacterianas $(1,1 \%)$ y 186 meningitis asépticas (10,1\%). En la evaluación inicial se detectaron 155 pacientes con meningitis $(155 / 1.774=8,7 \%)$ y en la reevaluación (media de 39,5 \pm 25,2 horas más tarde) se diagnosticaron 51 casos más (51/ $135=37,8 \%)$. Conclusiones: La meningitis fue un diagnóstico frecuente en recién nacidos febriles sin signos de focalización, predominando la meningitis aséptica. En casi $25 \%$ de los casos se llegó a este diagnóstico mediante la ejecución de la punción lumbar al ser re-evaluados.

\section{Referencias}

1.- Selbst S M, Korin J B. Malpractice and emergency care: Doing right by the patientand yourself. Contemporary Pediatr 2000; 17: 88-106.

2.- Bonadio W A. Evaluation and management of serious bacterial infections in neonates. $\mathrm{J}$ Pediatr 1990; 9: 905-12.

3.- Kneen R, Solomon T, Appleton R. The role of lumbar puncture in children with suspected central nervous system infection.
BMC Pediatrics 2002; 2: 8.

4.- Gorelick P B, Biller J. Lumbar puncture. Technique, indications, and complications. Postgrad Med 1986; 79: 257-68.

5.- Díaz Álvarez M, Fernández M T, MorenoVázquez O. Protocolo de atención del recién nacido febril sin signos de focalización. Rev Cubana Pediatr 1997; 69: 169-78.

6.- Díaz Álvarez M, Moreno Vázquez O, Fernández de la Paz M T, Martínez Canalejo H. Nuevos criterios de bajo riesgo de infección bacteriana severa en recién nacidos febriles. Rev Cubana Pediatr 1996; 68: 77-84.

7.- Kadish H A, Loveridge B, Tobey J, Bolte R G, Corneli H M. Applying outpatient protocols in febrile infants 1-28 days of age: can the threshold be lowered? Clin Pediatr (Phila) 2000; 39: 81-8.

8.- Chiu C H, Lin T Y, Bullard M J. Application of criteria identifying febrile outpatient neonates at low risk for bacterial infections. Pediatr Infect Dis J 1994; 13: 946-9.

9.- Baker M D, Bell L M, Avner J R. The 
efficacy of routine outpatient management without antibiotics of fever in selected infants. Pediatrics 1999; 103: 627-31.

10.- Berkowitz C D, Uchiyama N, Tully S B, Marble R D, Spencer M, Stein M T, et al. Fever in infants less than two months of age: Spectrum of disease and predictors of outcome. Pediatr Emerg Med 1985; 1: 128-35.

11.- Pantell R H, Newman T B, Bernzweig J, Bergman D A, Takayama J I, Segal M, et al. Management and outcomes of care of fever in early infancy. JAMA 2004; 291: 1203-12.

12.- Stanley R, Pagon Z, Bachur R. Hyperpyrexia among infants younger than 3 months. Pediatr Emerg Med 2005; 21: 291.

13.- Baraff L J, Bass J W, Fleisher G R, Klein J O, McCracken G H Jr, Powell K R, et al. Practice guideline for the management of infants and children 0 to 36 months of age with fever without source. Pediatrics 1993; 92: $1-12$

14.- Malbon K, Mohan R, Nicholl R. Should a neonate with possible late onset infection always have a lumbar puncture? Arch Dis Child 2006; 91: 75-6.

15.- Wilhelm S, Kelsberg G, Safranek S, Ward R. Clinical inquiries. Which infants need lumbar puncture for suspected sepsis? J Fam Pract 2003; 52: 806-8.
16.- Cincinnati Children’s Hospital Medical Center. Evidence based clinical protocol guideline for fever of uncertain source in infants 60 days of age or less. Cincinnati, Ohio: Cincinnati Children's Hospital Medical Center; 1998. URL disponible en: www.guideline.gov/summary/summary. aspx?doc_id=4130\&nbr=3166 [accedido 4 octubre 2006].

17.- Madigan Army Medical Center (MAMC) Referral Guidelines. Clinical standard for management and evaluation of infants $<90$ days of age with fever without an evident source.[en línea] 2003 URL disponible en: http://www.mamc.amedd.army.mil/Clinical/ standards/febrile_infant_cor.htm [accedido enero 2006].

18.- Steere M, Sharieff GQ, Stenklyft PH. Fever in children less than 36 months of age questions and strategies for management in the emergency department. J Emerg Med 2003; 25: 149-57.

19.- Brook I: Unexplained fever in young children: how to manage severe bacterial infection. Br Med J 2003; 327: 1094-5.

20.- Bonsu B K, Harper M B. Utility of the peripheral blood white blood cell count for identifying sick young infants who need lumbar puncture. Ann Emerg Med 2003; 41: 206-14.

21.- Sehabiague G, Bello O. Fiebre sin foco. Arch
Pediatr Urug 2001; 72 (S): S60-S61.

22.- Colectivo de autores: Atención del Recién Nacido Febril sin Signos de Focalización. En: Guías de Prácticas Clínicas en Neonatología. La Habana, 1999: Editorial Ciencias Médicas. p. 305-12.

23.- Oray-Schrom P, Phoenix C, St. Martin D, Amoateng-Adjepong Y. Sepsis workup in febrile infants 0-90 days of age with respiratory syncytial virus infection. Pediatr Emerg Care 2003; 19: 314-9.

24.- Brik R, Hamissah R, Shehada N, Berant M. Evaluation of febrile infants under 3 months of age: is routine lumbar puncture warranted? Isr J Med Sci 1997; 33: 93-7.

25.- Goldman R D, Matlow A, Linett L, Scolnik D: What is the risk of bacterial meningitis in infants who present to the emergency department with fever and pyuria? Can J Emerg Med 2003; 5: 394-9.

26.- Hoque M M, Ahmed A S, Chowdhury M A Darmstadt G L, Saha S K. Septicemic neonates without lumbar puncture: what are we missing? J Trop Pediatr 2006; 52: 63-5.

27.- Wiswell T E, Baumgart S, Gannon C M, Spitzer AR. Lumbar puncture in the evaluation for early neonatal sepsis [letter in reply]. Pediatrics 1996; 97(6 Pt 1): 930-1.

28.- Baraff L J. Management of fever without source in infants and children. Ann Emerg Med 2000; 36: 602-14. 